Classification

Physics Abstracts

$7.170-7.390-9.120$

\title{
ENTROPY OF SOLUBILITY OF SUBSTITUTIONAL IMPURITIES IN SOLID COMPOUNDS
}

\author{
F. BÉNIÈRE \\ Physique des Matériaux, Institut Universitaire de Technologie (Université de Rennes), \\ 22302 Lannion, France
}

(Reçu le 28 janvier 1976, accepté le 22 avril 1976)

\begin{abstract}
Résumé. - L'entropie vibrationnelle de solubilité, $\Delta S_{\text {sol }}$, est évaluée aux très faibles solubilités limites selon un calcul très simplifié. $\Delta S_{\text {sol }}$ est ainsi reliée simplement aux fréquences d'Einstein des solides purs. En outre, lorsque l'impureté introduit des lacunes de compensation de charge, $\Delta S_{\text {sol }}$ contient l'entropie de formation de lacune qui devient le terme prépondérant. Les résultats sont en accord raisonnable avec les quelques valeurs expérimentales disponibles.
\end{abstract}

Abstract. - The vibrational solubility entropy, $\Delta S_{\text {sol }}$, is evaluated for vanishingly low solid solubilities in a very simplified calculation. $\Delta S_{\text {sol }}$ is thus simply related to the Einstein frequencies of the pure solids. In addition, when the impurity introduces charge compensating vacancies, $\Delta S_{\text {sol }}$ includes the vacancy formation entropy, which becomes the predominating term. Results are in reasonable agreement with the few available experimental values.

1. Introduction. - The influence of impurities is known to play a major role on the physical properties of all solids : metals, semiconductors and ionic crystals. The solubility limit of any impurity in solid solution at a given temperature is ruled by thermodynamics [1]. Therefore, the solubility limit should be calculable from some thermodynamical parameters.

Let us consider a solid solution of $\mathrm{A}$ and $\mathrm{B}$ atoms in equilibrium with an excess of $\mathbf{B}$. We suppose that the $\mathrm{B}$ atoms are only lightly soluble in the A phase and we neglect the solubility of the A atoms in the B phase. So, A may be regarded as the solvent and B as the impurity (Fig. 1). The chemical potential of B is then the same in both phases :

$$
\mu_{\mathrm{B}(\mathbf{B})}^{0}=\mu_{\mathrm{B}(\mathrm{A})}=\mu_{\mathrm{B}(\mathrm{A})}^{0}+k T \ln a_{\mathrm{B}}
$$

where $a_{\mathrm{B}}$ is the activity of $\mathrm{B}$ and $\mu_{\mathrm{B}(\mathbf{A})}^{0}$ is the free enthalpy of one $\mathrm{B}$ atom in the solid solution $\mathrm{A}, \mathrm{B}$, excluding the mixing entropy. It contains two terms :

$$
\mu_{\mathbf{B}(\mathbf{A})}^{0}=\bar{H}_{\mathbf{B}(\mathbf{A})}-T \bar{S}_{\mathbf{B}(\mathbf{A})}
$$

$\bar{H}$ is the energy in the bonds of B with the surrounding atoms of the lattice (in a static state), per atom.

$T \bar{S}$ is the energy in the vibrations of the bonds of $\mathbf{B}$ with the lattice (dynamical state).

The configurational entropy $k \ln a_{\mathrm{B}}$ reduces to $k \ln s_{\mathbf{B}}\left(s_{\mathbf{B}}\right.$ is the solubility limit expressed as a mole fraction) when the $B$ atoms are distributed completely at random on the sites of A. Otherwise it is necessary to account for the ordering or the agglomeration by
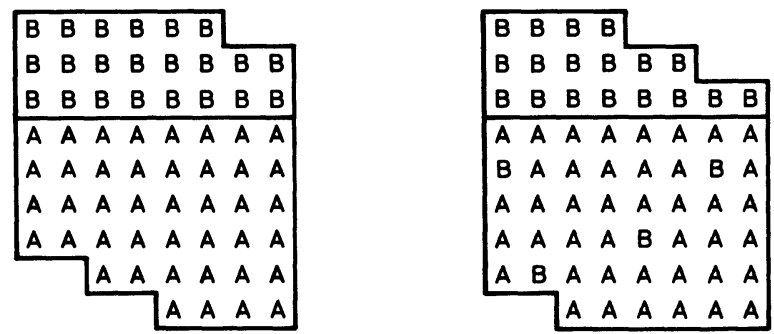

FIG. 1. - Schematic formation of the solid solution at the solubility limit. At thermodynamical equilibrium, $\mathrm{A}$ is saturated with $B$, an excess of $B$ is kept, while new cells of $A$ have been formed. A convenient method is to let $B$ to diffuse into $A$, which keeps $A$ as a single crystal. An accurate way to determine the solubility limit, and therefore $\Delta S_{\text {sol }}$, consists in labelling B with a radiotracer [7].

introducing an activity coefficient. Assuming first an ideal solution, the solubility limit from eq. (1) takes the form :

$$
s_{\mathrm{B}}=\exp \left(-\frac{\mu_{\mathrm{B}(\mathrm{A})}^{0}-\mu_{\mathrm{B}(\mathrm{B})}^{0}}{k T}\right)
$$

which defines the free enthalpy of solubility :

$$
\Delta G_{\text {sol }}=\mu_{\mathrm{B}(\mathrm{A})}^{0}-\mu_{\mathrm{B}(\mathrm{B})}^{0}=\Delta H_{\text {sol }}-T \Delta S_{\text {sol }} .
$$

According to eqs. (2) and (4), the enthalpy of solubility is :

$$
\Delta H_{\text {sol }}=\bar{H}_{\mathbf{B}(\mathbf{A})}-\bar{H}_{\mathbf{B}(\mathbf{B})}
$$


which accounts for the difference of energy of the bonds $\mathrm{A}-\mathrm{B}$ and $\mathrm{B}-\mathrm{B}$, while the entropy of solubility :

$$
\Delta S_{\text {sol }}=\bar{S}_{\mathrm{B}(\mathbf{A})}-\bar{S}_{\mathrm{B}(\mathbf{B})}
$$

is the vibrational entropy change when $B$ is surrounded by A or B atoms, respectively.

We try in the present paper to estimate this solubility entropy. For the further purpose of comparison with experiment, we first review the experimental methods leading to $\Delta S_{\text {sol }}$.

2. Survey of experimental methods and results. 2.1 SPECIFIC HEATS. - The most direct way would be to measure the specific heat from $0 \mathrm{~K}$ up to the temperature $T$ for the three solids : A, B and solid solution $\mathrm{AB}$.

$\Delta S_{\text {sol }}$ would then be obtained from Nernst law :

$$
S=\int_{0}^{T} \frac{C_{\mathrm{p}} \cdot \mathrm{d} T}{T} .
$$

However, the Nernst principle does not apply for a solid solution because it is not possible to keep the solid solution in a frozen state very far below the demixing temperature.

2.2 Heat Of Formation. - Barrett and Wallace [2] measured the heat of demixing (equal to $-\Delta H_{\text {sol }}$ ) of the solid solution $\mathrm{NaCl}, \mathrm{KCl}$ by a calorimetric method. This gives at thermal equilibrium :

$$
\Delta H_{\text {sol }}=T\left(\Delta S_{\text {sol }}+S_{\text {conf }}\right)
$$

i.e., the sum of the solubility entropy and of the configurational entropy. Unfortunately, the latter generally differs significantly from the entropy of random mixing, especially in the concentration range of practical use for this method, what limits its interest.

2.3 Elastic CONSTANTS. - The vibrational entropy has been previously expressed as a function of the Debye temperature of the solid solution, itself expressed as a function of the elastic constants [3]. Again, this concerns only high solubilities and, actually, the method was applied for predicting exsolution domes [4].

2.4 Solubility Limit. - The solubility limit is measured by various techniques, such as X-ray diffraction, which gives the $\Delta G_{\text {sol }}$ derived from eqs. (3) and (4) at a number of temperatures. Again, those methods apply for rather large solubilities. Then the surrounding of the impurity changes as $T=\varphi\left(s_{\mathrm{B}}\right)$ varies, entailing a strong dependence of $\Delta H_{\text {sol }}$ and $\Delta S_{\text {sol }}$ on temperature. In fact, it is only for very low solubility limits that a linear dependence of $\Delta G_{\text {sol }}$ on $T$ is observed, giving the limiting values for $\Delta S_{\text {sol }}$ and $\Delta H_{\text {sol }}$.

Solubilities as low as $10^{-3}$ and less can be easily determined thanks to the sensitivity of the radiotracer technique, as successfully applied in a few systems : $\mathrm{NaCl}, \mathrm{YCl}_{3}$ [5], $\mathrm{KCl}, \mathrm{MnCl}_{2}$ [6] and $\mathrm{KCl}, \mathrm{K}_{2} \mathrm{SO}_{4}$ [7]. In those cases the solubility limit is reached by letting excess impurity diffuse into the pure solvent perfect crystal (Fig. 1). The solubility limit can be reached even for a low diffusion coefficient because the latter is enhanced by a self-doping effect. Thus, the ionic crystals will be taken as an example of the following estimation of the solubility entropy.

3. Calculation of the solubility entropy. - The analysis will be restricted to the following range of concentration and temperature :

- The impurity is surrounded only by atoms of the host lattice. This implies low solubilities.

- The entropy is calculated for solubility limit temperatures higher than the Einstein temperature in the harmonic approximation. The vibrational entropy change when the pulsations change from $\omega_{i}$ to $\omega_{i}^{\prime}$ is then given by :

$$
\Delta S=k \sum_{i} \ln \left(\omega_{i}^{\prime} / \omega_{i}\right) .
$$

We follow in that way the calculation of the phase change entropy of Friedel [8] and of the vacancy formation entropy of Dobrzynski [9] and ourself [10].

3.1 SOLUBILITY ENTROPY OF AN IMPURITY OF SAME VALENCY AS THE SUBSTITUTED ION. - This is the case of, e.g., the solubility of $\mathrm{KCl}$ in $\mathrm{NaCl}$.

According to eq. (4) and making use of the additivity rule to express the chemical potentials of the ionic species, one gets :

$$
\begin{aligned}
& \Delta G_{\mathrm{sol}}=\mu_{\mathrm{K}^{+}(\mathrm{NaCl})}^{0}-\mu_{\mathrm{K}^{+}(\mathrm{KCl})}^{0}+ \\
& +\mu_{\mathrm{Cl}^{-}(\mathrm{NaCl})}^{0}-\mu_{\mathrm{Cl}^{-}(\mathrm{KCl})}^{0} .
\end{aligned}
$$

From this equation and equation (6) one obtains the solubility entropy in the form :

$$
\begin{aligned}
& \Delta S_{\mathrm{sol}}=\bar{S}_{\mathrm{K}^{+}(\mathrm{NaCl})}-\bar{S}_{\mathrm{K}^{+}(\mathrm{KCl})}+ \\
& +\bar{S}_{\mathrm{Cl}^{-}(\mathrm{NaCl})}-\bar{S}_{\mathrm{Cl}^{-}(\mathrm{KCl})} .
\end{aligned}
$$

This expression can be calculated as a function of the vibration frequencies through the fundamental eq. (8). A solid compound such as $\mathrm{NaCl}$, containing $N$ molecules, is to be regarded in the Einstein model as $3 N$ independent oscillators of pulsation $\omega_{\mathrm{Na}^{+}}$ and $3 N$ independent oscillators of pulsation $\omega_{\mathrm{Cl}^{-}}$. The relevant pulsations are given by the equations :

$$
\begin{aligned}
& \omega_{\mathrm{Na}^{+}(\mathrm{NaCl})}=\left(\alpha_{\mathrm{NaCl}} / m_{\mathrm{Na}^{+}}\right)^{1 / 2} \\
& \omega_{\mathrm{Cl}{ }^{-}(\mathrm{NaCl})}=\left(\alpha_{\mathrm{NaCl}} / m_{\mathrm{Cl}^{-}}\right)^{1 / 2}
\end{aligned}
$$

where $\alpha$ is the force constant and $m$ the ion mass.

Furthermore, when an ion is substituted for a foreign ion, Lannoo and Dobrzynski [11] showed 
that a similar equation for the pulsation of the foreign ion, e.g. $\mathrm{K}^{+}$in $\mathrm{NaCl}$ :

$$
\omega_{\mathrm{K}^{+}(\mathrm{NaCl})}=\left(\alpha^{\prime} / m_{\mathrm{K}^{+}}\right)^{1 / 2}
$$

holds true with a good accuracy with the perturbed force constant, $\alpha^{\prime}$, essentially depending on the interaction between $\mathrm{K}^{+}$and the (six) $\mathrm{Cl}^{-}$at first neighbour sites. Since the configuration of the immediate surrounding is the same for $\mathrm{K}^{+}$in $\mathrm{KCl}$ and $\mathrm{K}^{+}$ in $\mathrm{NaCl}$, we thus assume that $\alpha^{\prime} \simeq \alpha_{\mathrm{KCl}}$. It follows that :

$$
\bar{S}_{\mathrm{K}^{+}(\mathrm{NaCl})}-\bar{S}_{\mathrm{K}^{+}(\mathrm{KCl})}=3 k \ln \frac{\omega_{\mathrm{K}^{+}(\mathrm{KCl})}}{\omega_{\mathrm{K}^{+}(\mathrm{NaCl})}} \simeq 0 .
$$

Combining the eqs. (8) to (14), we may write the final result :

$$
\Delta S_{\mathrm{sol}}=3 k \ln \frac{\omega_{\mathrm{Cl}^{-}(\mathrm{KCl})}}{\omega_{\mathrm{Cl}-(\mathrm{NaCl})}}
$$

We shall now proceed to the numerical evaluation by following two quite distinct approaches.

3.1.1 Specific heat method. - The two Einstein frequencies of the binary compound must satisfy the Einstein equation of the vibrational energy $E$ :

$$
\begin{aligned}
E= & \int_{0}^{T} C_{\mathrm{v}} \mathrm{d} T=3 N \hbar \times \\
& \times\left[\frac{\omega_{\mathrm{Cl}^{-}}}{\exp \left(\hbar \omega_{\mathrm{Cl}^{-}} / k T\right)-1}+\frac{\omega_{\mathrm{Na}^{+}}}{\exp \left(\hbar \omega_{\mathrm{Na}^{+}} / k T\right)-1}\right] .
\end{aligned}
$$

Instead of integrating the specific heat $C_{\mathrm{v}}$, it is more convenient to make use of the already computed Debye temperature $\theta$, and to identify the preceding equation to the following expression of the energy :

$$
E=18 N k T\left(\frac{T}{\theta}\right)^{3} \int_{0}^{\theta / T} \frac{x^{3}}{\mathrm{e}^{x}-1} \mathrm{~d} x .
$$

Substituting for $\omega_{\mathrm{Na}^{+}}$from eqs. (11) and (12), one readily obtains $\omega_{\mathrm{Cl}^{-}(\mathrm{NaCl})}$ (and $\left.\omega_{\mathrm{Cl}^{-}(\mathrm{KCl})}\right)$. However, the result can depend on the temperature $T$ chosen when equating (16) with (17) and a relatively high temperature of the order of the Debye temperature is to be taken.

With the available data for the masses of $\mathrm{Na}^{+}, \mathrm{K}^{+}$ and $\mathrm{Cl}^{-}$and the Debye temperatures of $\mathrm{NaCl}$ and $\mathrm{KCl}$ [12], one obtains :

$$
\begin{aligned}
& \omega_{\mathrm{Cl}^{-}(\mathrm{NaCl})}=3.18 \times 10^{13} \mathrm{rad} . \mathrm{s}^{-1} \\
& \omega_{\mathrm{Cl}^{-}(\mathrm{KCl})}=2.94 \times 10^{13} \mathrm{rad} . \mathrm{s}^{-1}
\end{aligned}
$$

and whence from [15] :

$$
\Delta S_{\mathrm{sol}} \sim-0.2 \mathrm{k} \text {. }
$$

3.1.2 Bulk modulus method. - It is also possible to evaluate the vibration frequencies by calculating the force constants from the second derivative of the potential energy. This gives [11] as a function of $a$, the anion-cation distance, and $B$, the bulk modulus :

$$
\alpha=6 a B \text {. }
$$

With the available data for $a, m$ and $B$ [13], one gets from (12) :

$$
\omega=(6 a B / m)^{1 / 2}
$$

the numerical results :

$$
\begin{aligned}
\omega_{\mathrm{Cl}^{-}(\mathrm{NaCl})} & =2.62 \times 10^{13} \mathrm{rad} . \mathrm{s}^{-1} \\
\omega_{\mathrm{Cl}^{-}(\mathrm{KCl})} & =2.36 \times 10^{13} \mathrm{rad} . \mathrm{s}^{-1}
\end{aligned}
$$

and finally a value for $\Delta S_{\text {sol }}$ in satisfactory agreement with the previous one, thus we feel justified in comparing our approach with experimental results.

3.1.3. Comparison with experiment. - Barrett and Wallace [2] reported some values of the total entropy of solubility which contains the configuration entropy. If for the latter one takes the entropy of random mixing (e.g. $0.3 \mathrm{k}$ for a solid solution of $10 \% \mathrm{KCl}$ ) one obtains for the (vibration) solubility entropy a value close to $0 \mathrm{k}$.

Compared with the previous calculated value one can conclude that the right order of magnitude is found. A more direct experimental result is needed for confirmation.

3.1.4 Important remark. - From eq. (15) one obtains at once the entropy of the solubility of $\mathrm{NaCl}$ in $\mathrm{KCl}$ which is the inverse of the solubility entropy of $\mathrm{KCl}$ in $\mathrm{NaCl}$. Let us compare both solubilities at the same temperature, given as the intersections of an horizontal line with the demixing curve in the phase diagram :

$$
\frac{s_{\mathrm{NaCl}(\mathrm{KCl})}}{s_{\mathrm{KCl}(\mathrm{NaCl})}}=A \exp \left(\frac{\Delta S_{\mathrm{sol.NaCl}}-\Delta S_{\mathrm{sol.KCl}}}{k}\right)
$$

where $A$ is the enthalpy term. Substituting for the $\Delta S_{\text {sol }}$ from (15) this equation becomes very much simplified :

$$
\frac{s_{\mathrm{NaCl}(\mathrm{KCl})}}{s_{\mathrm{KCl}(\mathrm{NaCl})}}=A\left(\frac{\omega_{\mathrm{Cl}^{-}(\mathrm{NaCl})}}{\omega_{\mathrm{Cl}^{-}(\mathrm{KCl})}}\right)^{6} \sim 1.7 A .
$$

Besides the influence of the enthalpy term, we find therefore an entropy term higher than unity, which may partly account for the experimental fact that $\mathrm{NaCl}$ is more soluble in $\mathrm{KCl}$ than the inverse.

Therefore eq. (15) implies that all other things being equal the most soluble species is that having the highest Debye temperature. Since in those systems such as $\mathrm{KCl}-\mathrm{KBr}$ or $\mathrm{AgCl}-\mathrm{AgBr}$ the highest Debye temperature is observed for the small ion species, this is equivalent to the well-known general rule all other things being equal it is more difficult to replace a small 


\section{TABLE I}

Vibrational entropy of solubility in alkali halides. The experimental values result from tracer techniques [5-7], calorimetry [2], and conductivity data [15-16]. When vacancies are created, the calculated values include only the vacancy formation entropy from reference [10]

$\begin{array}{cccccc}\text { Formation of } & \begin{array}{c}\mathrm{No} \text { vacancy } \\ \mathrm{KCl} \text { in } \mathrm{NaCl}\end{array} & \begin{array}{c}\text { One cation vacancy } \\ \mathrm{MnCl}_{2} \text { in } \mathrm{KCl}\end{array} & \begin{array}{c}\text { One cation vacancy } \\ \mathrm{CdCl}_{2} \text { in } \mathrm{NaCl}\end{array} & \begin{array}{c}\text { One anion vacancy } \\ \mathrm{K}_{2} \mathrm{SO}_{4} \text { in } \mathrm{KCl}\end{array} & \begin{array}{c}\text { Two cation vacancies } \\ \mathrm{YCl}_{3} \text { in } \mathrm{NaCl}^{-}\end{array} \\ \begin{array}{c}\Delta S_{\text {sol }} / k \\ \text { experimental }\end{array} & \sim 0[2] & 4.2[6] & 1.1[15]-14[16] & 6.9[7] & 13[5] \\ \begin{array}{c}\Delta S_{\text {sol }} / k \\ \text { calculated }\end{array} & -0.5 & 5 \pm 1 & 5 \pm 1 & 5 \pm 1 & 10 \pm 2\end{array}$

ion in the lattice with a large one than to do the reverse. Eq. (15) could thus be considered as a partial demonstration of this rule.

This seems quite general and to concern also the metal. As an example, the solubility curve of $\mathrm{Sn}-\mathrm{Pb}$ is very unsymmetrical with a much larger solubility of Sn. Actually, the Debye temperature of $\mathrm{Sn}$ is much larger than that of $\mathrm{Pb}$.

3.2 SOLUBILITY ENTROPY OF IMPURITIES INTRODUCING ONE OR MORE VACANCIES. - As an example, the divalent impurity $\mathrm{Sr}^{++}$is incorporated in the $\mathrm{NaCl}$ lattice as one $\mathrm{SrCl}_{2}$ molecule replacing two $\mathrm{NaCl}$ molecules. So, a cation vacancy is created. $\mathrm{The}^{\mathrm{Sr}^{++}}$ impurities and the vacancies partly associate to give impurity-vacancy complexes. But, the particular thermodynamical equilibrium constituted by both ends of the successive reaction :

$$
\begin{aligned}
\mathrm{SrCl}_{2} \text { (crystal) } & \rightleftarrows \text { impurity-vacancy complex in } \mathrm{NaCl} \\
& \rightleftarrows \text { free isolated } \mathrm{Sr}^{++}, \mathrm{Cl}^{-} \text {and vacancy }
\end{aligned}
$$

only involves :

- the non-associated $\mathrm{Sr}^{++}$, of mole fraction $s$. Through suitable transport process measurements, $s$ can be determined from $C$, the total mole fraction of free and associated impurities, and $p$, the association degree since $s=C(1-p)$,

- the free vacancies, whose entropy and enthalpy of formation are well known from the pure crystal results.

In terms of chemical potentials, this equilibrium implies that :

$$
\mu_{\mathrm{SrCl}_{2}\left(\mathrm{SrCl}_{2}\right)}^{0}=\mu_{\mathrm{Sr}^{+}+(\mathrm{NaCl})}+2 \mu_{\mathrm{Cl}^{-}(\mathrm{NaCl})}+\mu_{\mathrm{v}}
$$

where $\mu_{\mathrm{v}}$ is the chemical potential of the free cation vacancies, of mole fraction equal to $x$. This equation can be developed into the following form :

$$
\begin{aligned}
& \mu_{\mathrm{Sr}^{++}\left(\mathrm{SrCl}_{2}\right)}^{0}+2 \mu_{\mathrm{Cl}^{-}\left(\mathrm{SrCl}_{2}\right)}^{0}=\mu_{\mathrm{Sr}^{++}\left(\mathrm{NaCl}^{2}\right)}^{0} \\
& \quad+R T \ln s+2 \mu_{\mathrm{Cl}^{-}(\mathrm{NaCl})}^{0}+\mu_{\mathrm{v}}^{0}+R T \ln x .
\end{aligned}
$$

Rearranging of the terms yields :

$$
x s=\exp \left(-\Delta G_{\text {sol }} / k T\right) .
$$

We need express only the entropy :

$$
\begin{aligned}
\Delta S_{\mathrm{sol}}=[ & \bar{S}_{\mathrm{Sr}^{++}}(\mathrm{NaCl}) \\
& -2\left[\bar{S}_{\mathrm{Sr}^{-}(\mathrm{NaCl})}-\bar{S}_{\mathrm{Cl}^{-}\left(\mathrm{SrCl}_{2}\right)}\right]+S_{\mathrm{v}} .
\end{aligned}
$$

The entropy of formation of a single cation vacancy cannot be reached experimentally. Nevertheless, $S_{\mathrm{s}}$ entropy of formation of a Schottky pair (isolated cation and anion vacancies) is fairly well known [10] and for an estimate we assume :

$$
S_{\mathrm{v}} \sim S_{\mathrm{s}} / 2 .
$$

Although the terms in brackets are difficult to evaluate because the $\mathrm{NaCl}$ and $\mathrm{SrCl}_{2}$ lattices are different, a rough analysis on the basis of the Einstein pulsations shows that the contribution of the vacancy should be the predominating term, so that for any divalent ion :

$$
\Delta S_{\mathrm{sol}} \sim S_{\mathrm{s}} / 2
$$

It is straightforward to apply this procedure to the case when more than one vacancy are created, for example the solubility of $\mathrm{YCl}_{3}$ in $\mathrm{NaCl}$. One thus obtains for any trivalent ion :

$$
\Delta S_{\mathrm{sol}} \sim S_{\mathrm{s}}
$$

Comparison with experiment. - Some results are reported in table I when one anion vacancy, one cation vacancy and two cation vacancies respectively are created. At least, the right order of magnitude is found. It is difficult to go further because of the lack of experimental data. This is emphasized by the discrepancy between the values reported for the same system $\mathrm{CdCl}_{2}(\mathrm{NaCl})$ though they result from two similar, independent and recent analyses of conductivity data in doped crystals $[15,16]$.

4. Conclusion. - By assuming rather crude approximations, the solubility entropy can be related to a few thermodynamical parameters of the pure solids. This analysis could be somewhat improved by extending 
the influence of the impurity [14] and also by accounting for local ordering when observed (configurational entropy over the random mixing entropy).

Predicting of solid solubility limits would need - in addition to the entropy term - knowledge of the enthalpy term $\Delta H_{\text {sol }}$, which seems feasible on the usual line [1]. At last, the simultaneous determination of $\Delta H_{\text {sol }}$ and $\Delta S_{\text {sol }}$ would also lead to the configurational entropy through eq. (7).

Acknowledgments. - I would like to acknowledge my indebtedness to Professor J. Friedel for his interest and considerable help to Dr L. Dobrzynski, and to Professor M. A. Nusimovici for fruitful discussions.

\section{References}

[1] Among many good articles about thermodynamics of lattice defects : Howard, R. E. and Lidiard, A. B., Rep. Prog. Phys. 27 (1964) 161.

Franklin, A. D., Point Defects in Solids, Crawford and Slifkin eds. (Plenum Press, New York) 1 (1972) 1

[2] Barrett, W. T. and Wallace, W. E., J. Am. Chem. Soc. 76 (1954) 366.

[3] Kleppa, O. J., Metallic Solid Solutions, Friedel and Guinier eds. (Benjamin, New York) 1963.

[4] Fancher, D. L. and Barsch, G. R., J. Phys. \& Chem. Solids 30 (1969) 2517.

[5] Bénière, F. and Rokbani, R., J. Phys. \& Chem. Solids 36 (1975) 1151 .

[6] Brun, A., Dansas, P. and Bénière, F., J. Phys. \& Chem. Solids 35 (1974) 249.

[7] Bénière, M., BÉNiÈre, F. and Chemla, M., Solid State Commun. 13 (1973) 1339.
[8] Friedel, J., J. Physique Lett. 35 (1974) L-59.

[9] Dobrzynski, L., J. Phys. \& Chem. Solids 30 (1969) 2395.

[10] BÉNière, F., J. Physique Lett. 36 (1975) L-9.

[11] Lannoo, M. and Dobrzynski, L., J. Phys. \& Chem. Solids 33 (1972) 1447.

[12] SeITZ, F., Théorie moderne des solides (Masson ed.) 1949, p. 121.

[13] Kittel, C., Introduction to Solid State Physics (Wiley ed.) 1971, p. 121.

[14] Mahanty, J. and Sachdev, M., J. Phys. C. Solid St. Phys. 3 (1970) 773.

[15] Chapman, J. A. and Lilley, E., J. Physique Colloq. 34 (1973) C9-455.

[16] Laredo, E. and Figueroa, D., J. Physique Colloq. 34 (1973) C9-449. 